\title{
Superimposition of maxillary digital models using the palatal rugae: Does ageing affect the reliability?
}

\author{
Daniela Garib $^{1}$ (D) | Felicia Miranda ${ }^{2}$ | Marilia S. Yatabe ${ }^{3}$ (D) | \\ José Roberto Pereira Lauris ${ }^{4}$ | Camila Massaro ${ }^{2}$ | James A. McNamara Jr ${ }^{3}$ | \\ Hera Kim-Berman $^{3}$ | Guilherme Janson ${ }^{2}$ | Rolf G. Behrents ${ }^{5}$ | Lucia H. S. Cevidanes ${ }^{3}$ | \\ Antonio Carlos de Oliveira Ruellas ${ }^{3}$
}

\footnotetext{
${ }^{1}$ Department of Orthodontics, Bauru Dental School and Hospital for Rehabilitation of Craniofacial Anomalies, University of São Paulo, Bauru, Brazil

${ }^{2}$ Department of Orthodontics, Bauru Dental School, University of São Paulo, Bauru, Brazil

${ }^{3}$ Department of Orthodontics and Pediatric Dentistry, School of Dental Medicine, University of Michigan, Ann Arbor, MI, USA

${ }^{4}$ Department of Public Health, Bauru Dental School, University of São Paulo, Bauru, Brazil

${ }^{5}$ Orthodontic Program at the Center for Advanced Dental Education of Saint Louis University, Saint Louis, MO, USA

Correspondence

Dr. Daniela Garib, Department of Orthodontics, Bauru Dental School, University of São Paulo, Bauru, Brazil. Emails: dgarib@usp.br; dgarib@uol.com.br

Funding information

This work was supported by the São Paulo Research Foundation (FAPESP) (grant \#2016/11400-8).
}

\section{Structured Abstract}

Objectives: The aim of this study was to evaluate the reliability of 3-dimensional maxillary dental changes using two methods of digital model superimposition.

Setting and Sample Population: The Department of Orthodontics of Bauru Dental School, University of São Paulo and University of Michigan Craniofacial Growth Center. Fifteen subjects with normal occlusion.

Material \& Methods: The sample was composed of digital study models of 15 normal occlusion subjects taken at 13 (T1), 18 (T2) and 60 years of age (T3). Using the software SlicerCMF 3.1, superimposition (registration) was conducted using 9 landmarks placed on the incisive papilla, second and third palatal rugae and $10 \mathrm{~mm}$ distal to the third palatal rugae. Two registration methods were compared: landmarks (LA) and regions of interest (ROI). Three-dimensional changes of landmarks on the buccal cusp tip of posterior teeth bilaterally and the incisal edge of the right central incisor were measured by three examiners. Intraclass correlation coefficients and Bland-Altman method evaluated intra- and inter-examiner agreements.

Results: Good or excellent intra-examiner agreement was found for T1-T2 and T2-T3 measurements using both registration methods. Inter-examiner agreements were good to excellent for T1-T2 measurements and poor to fair for most T2-T3 measurements. Mean T1-T2 differences were less than $0.5 \mathrm{~mm}$ for most measurements.

Conclusion: Maxillary digital dental models of patients with normal occlusion superimposed on palatal rugae showed an adequate reliability for a 5-year interval comparison using landmarks or regions of interest. Lower than acceptable reproducibility using both superimposition methods was found for a 40-year interval comparison.

\section{KEYWORDS}

ageing, dental models, reproducibility of results 


\section{1 | INTRODUCTION}

The superimposition of digital dental models is an innovative tool to quantify individual dental changes between two time point records. ${ }^{1-5}$ Studies have shown that dental model superimposition is a reliable study method. ${ }^{2}$ The methodology for superimposing digital dental models, however, has not been fully standardized, as other commonly used orthodontic methods. ${ }^{6,7}$

The major challenge regarding dental model superimposition is selecting reliable and stable landmarks of reference to achieve a consistent technique. In the maxillary arch, the palatal rugae are considered a stable reference region, which has been used previously for superimposition. ${ }^{8-12}$ In order to evaluate the stability of the palatal rugae as reference area, palatal mini-implants have been used to superimpose models after orthodontic treatment. ${ }^{11}$ The medial points of the third palatal rugae and palatal shape were determined to be stable during treatment and were considered reliable reference landmarks for superimposition. ${ }^{11}$

A previous study used the palatal surface as reference for superimposition of dental models. ${ }^{4}$ This technique presented similar results for tooth movement compared to cephalometric analysis. ${ }^{4}$ Another study superimposed digital models using surface-to-surface matching technology. ${ }^{13}$ No differences in anterior, transverse and vertical tooth movements were found when comparing plaster and digital model superimposition. ${ }^{13}$ The authors concluded that digital dental model superimposition was accurate and reliable for the assessment of orthodontic tooth movements. ${ }^{13}$

The changes produced by ageing continue throughout adulthood. ${ }^{14}$ Longitudinal studies have followed the maturational processes in normal occlusions subjects until the fifth decade of life. ${ }^{15-18}$ A progressive reduction in arch width, arch length and maxillary arch perimeter was reported over the years in the maxillary arch. ${ }^{15,16,18}$ A reliable method of dental model superimposition would be useful to allow a more detailed observation of the individual three-dimensional displacement of each individual tooth during ageing.

Therefore, the aim of this study was to evaluate the reliability of 3-dimensional maxillary dental changes using two methods of digital dental model superimposition (landmarks and regions of interest) and two-time intervals ( 5 and 40 years). The hypotheses are that the two superimposition/registration methods show similar reliability at completion of pubertal growth and 40 years later.

\section{2 | MATERIAL AND METHODS}

This study was approved by the Institutional Review Board (Process number: 43931915.4.0000.5417). The sample was comprised of 15 subjects with normal occlusion from the Bauru Dental School, University of São Paulo $(n=10)$ and from the records of the University of Michigan Craniofacial Growth Center $(n=5)$. Written patient consents were obtained from the Bauru Dental School, University of São Paulo sample. Sample size calculation considered a power of $80 \%$, an alpha error of $5 \%$, a minimum difference to be detected of $1 \mathrm{~mm}$ and a standard deviation of $1.3 \mathrm{~mm}$ derived from a preliminary sample of 10 patients. A sample of 15 patients was necessary.

All subjects were in the complete permanent dentition and demonstrated Class I dental occlusions and well-balanced faces, absence of crossbites, normal overjet and overbite and a maximum $2 \mathrm{~mm}$ of incisor crowding with no previous history of orthodontic treatment. Plaster dental models taken at 13,18 and 60 years of age were scanned using a R700 3D Scanner (3Shape A/S, Copenhagen, Denmark). Impressions were performed with alginate, and plaster type 1 was used for all time points. The exclusion criteria were history of previous orthodontic treatment or tooth loss of 2 or more permanent teeth from T1 to T3, and absence of dental models at any of the 3 time points.

Maxillary digital models scanned and stored as.STL files were converted to.VTK meshes files, using SlicerCMF 3.1 software (www. slicer.org). The image analysis consisted of the following steps:

\section{$2.1 \mid$ Model orientation}

T1 dental models were oriented using the 3D coordinate system, using the transforms tool, of Slicer software (Figure 1). Using the maxillary occlusal perspective view, the midpalatal raphe was positioned coincident with the anteroposterior yellow line (sagittal plane). In the same view, the second palatal rugae were moved coincident to the superiorinferior green line (coronal plane). In the right side view, the occlusal plane, defined by passing through the maxillary first molar mesio-buccal cusp tip and maxillary canine cusp tip, was placed on the right-left red line (axial plane). On the model's frontal view, the cusp tips of the right and left canines were positioned on the red line (axial plane).

\section{2 | Model approximation}

Model approximation was conducted in two steps. a) The T2 maxillary digital dental model was approximated to the T1 model by placing 6 corresponding landmarks in the T2 and T1 3D digital dental models using the Slicer "CMF registration" tool. Dental landmarks approximation was performed as a first step in order to initially set the T2 model position. The landmarks were placed on the tip of the

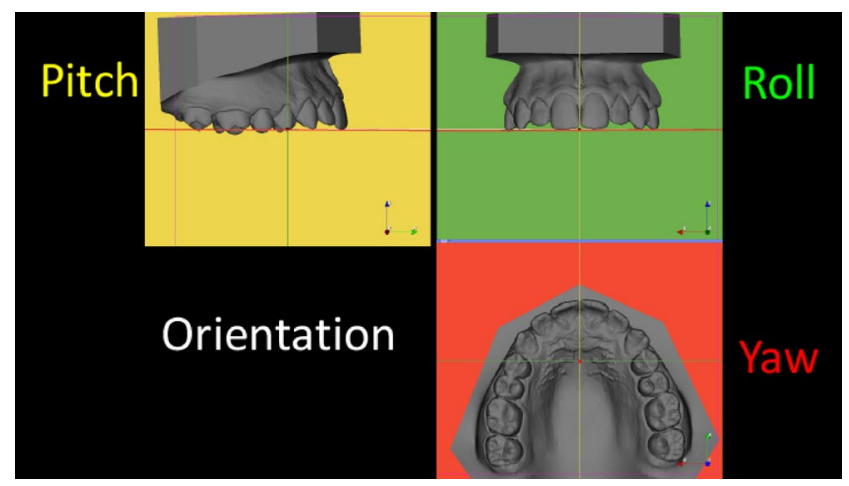

FIGURE 1 Model orientation [Colour figure can be viewed at wileyonlinelibrary.com] 


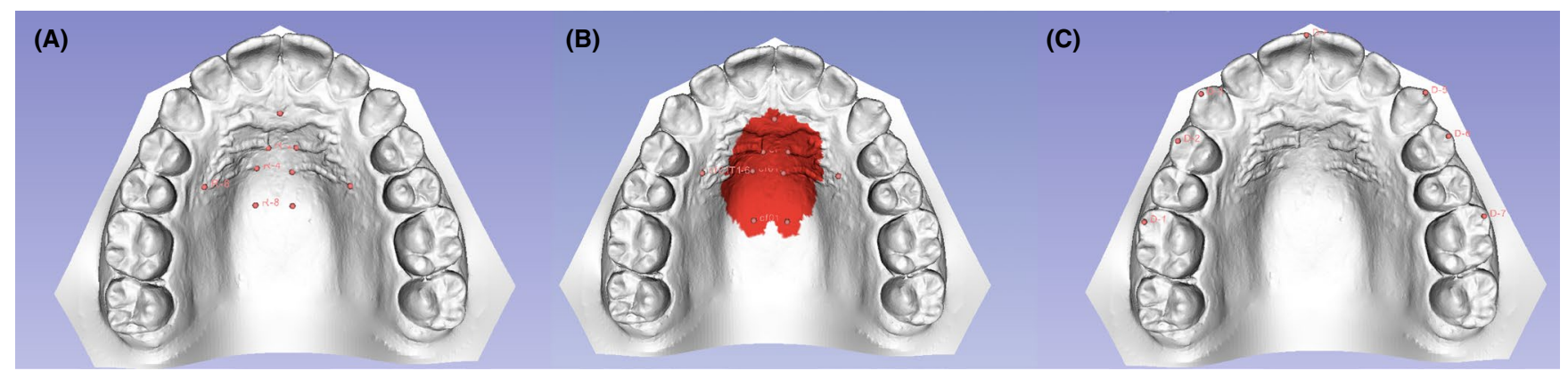

FIG URE 2 A, Nine landmarks were used for landmark registration on the palatal rugae. B, Region of interest around the nine landmarks used for ROI registration. C, Landmarks used for tridimensional quantitative measurements [Colour figure can be viewed at wileyonlinelibrary.com]

mesio-buccal cusp of the first molar, the buccal cusp of the first premolar and in the tips of the cusp of the right and left canines. All landmarks were placed bilaterally. The Q3DC tool of the SlicerCMF 3.1 software displayed the $\mathrm{x}, \mathrm{y}$ and $\mathrm{z}$ coordinates for each landmark. Using the $x, y$ and $z$ coordinates of T1 as reference, the software changed the spatial position of $\mathrm{T} 2$ to match the T1 coordinates. As a result, the T2 and T1 maxillary models were approximated by superimposition of the corresponding landmarks. b) Using the transform tool, the palatal rugae of the T2 maxillary dental model with $50 \%$ transparency were fine-tuned by manual approximation to the palatal rugae of the oriented T1 model.

\section{3 | Superimposition using Landmarks (LA)}

Corresponding landmarks then were placed at the posterior limit of the incisive papilla, at the medial edges of the second palatal rugae and at the medial and lateral edges of the third rugae (Figure 2). Two additional landmarks were projected $10 \mathrm{~mm}$ distal to the medial edge of third rugae landmarks using the transforms tool (Figure 2). The Slicer "CMF registration" tool then registered the T2 maxillary model relative to the oriented $\mathrm{T} 1$ model by matching the coordinates of the corresponding landmarks (Figure 3).

\section{4 | Superimposition using Regions of Interest (ROI)}

The same 9 landmarks described at step 3 were used to define regions of interest (Figure 2). The dimension of each ROI was defined as the radius of 5 vertices of the model meshes for the incisive papilla and the third rugae projection landmarks. The medial edges of the second and third rugae were assigned with ROls of radius of 20 and 15, respectively. The lateral edge of third rugae had ROI of 1 in order to avoid extending on the tooth region. The Slicer "CMF registration" tool then registered the T2 maxillary model relative to the oriented T1 model by matching the coordinates of the corresponding regions of interest.

Steps 2 to 4 were repeated for the T3 maxillary digital models relative to the registered $\mathrm{T} 2$ models.

\section{5 | Three-dimensional quantitative measurements}

Using the Q3DC tool of the Slicer software, landmarks were placed in T1, registered T2 and registered T3 models at the tip of the mesiobuccal cusp of the first molars, buccal cusp of the first premolars and canines bilaterally (Figure 2). A seventh landmark was placed on the mesial angle of the incisal edge of the right central incisor. Differences between the 2 time points (T1 to T2 and T2 to T3) were measured considering the 3D Euclidian distance displacement and also the changes in the coordinates $x, y$, and z. Forward, inferior and lateral displacements had positive values. Backward, superior and medial displacements had negative values.

\section{6 | Statistical analyses}

Statistical analysis was carried out using SPSS Statistical Software Package (version 21.0; SPSS, Chicago, IL). All above-mentioned steps were performed by three examiners. The first examiner repeated the steps twice with a minimum 15-day interval. Descriptive statistics included the mean and standard deviation of three-dimensional displacements from T1 to T2 comprising a 5-year interval and from T2 to T3 comprising a 42-year interval. Intra- and
FIGURE 3 T2 models registered on T1 model using points of interest. LeftFrontal view. Right-Lateral view [Colour figure can be viewed at wileyonlinelibrary. com]

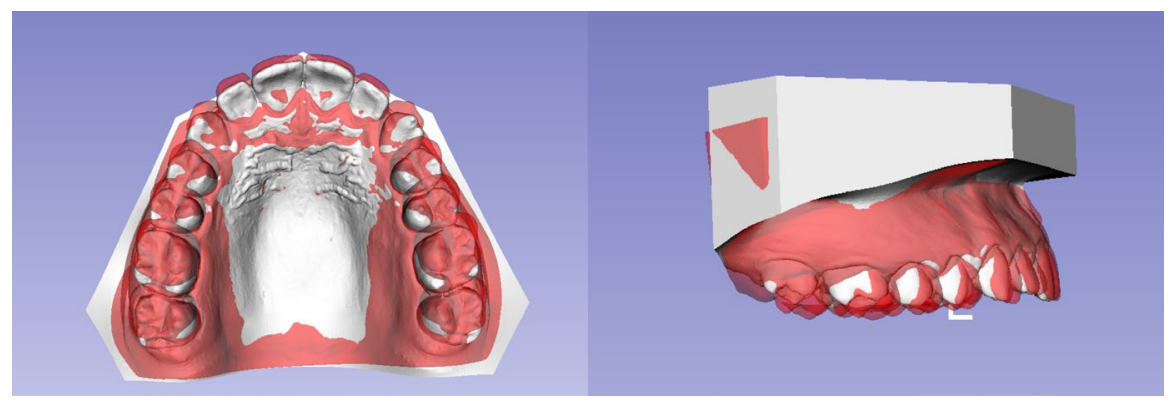


TAB LE 1 Measurement agreements using dental model registration on landmarks (LA) and regions of interest (ROI) (Intraclass correlation coefficient)

\begin{tabular}{|c|c|c|c|c|c|c|c|c|c|c|}
\hline & & \multirow[b]{2}{*}{ Measurement } & \multicolumn{2}{|c|}{$\begin{array}{l}\text { CHANGES LA } \\
(\mathrm{mm})\end{array}$} & \multicolumn{2}{|c|}{$\begin{array}{l}\text { CHANGES ROI } \\
(\mathrm{mm})\end{array}$} & \multirow{2}{*}{$\begin{array}{l}\text { Intra-exam- } \\
\text { iner } \\
\text { agreement } \\
\text { LA }\end{array}$} & \multirow{2}{*}{$\begin{array}{l}\text { Inter-ex- } \\
\text { aminer } \\
\text { agreement } \\
\text { LA }\end{array}$} & \multirow{2}{*}{$\begin{array}{l}\text { Intra-exam- } \\
\text { iner } \\
\text { agreement } \\
\text { ROI }\end{array}$} & \multirow{2}{*}{$\begin{array}{l}\text { Inter-exam- } \\
\text { iner } \\
\text { agreement } \\
\text { ROI }\end{array}$} \\
\hline & & & Mean & SD & Mean & SD & & & & \\
\hline \multirow[t]{18}{*}{ T1-T2 } & \multirow{3}{*}{$\begin{array}{l}\text { Right first } \\
\text { molar }\end{array}$} & $R-L$ & 0.081 & 0.742 & 0.079 & 0.691 & 0.973 & 0.832 & 0.935 & 0.798 \\
\hline & & $A-P$ & 1.704 & 0.956 & 1.690 & 1.000 & - & 0.659 & 0.946 & 0.733 \\
\hline & & S-I & -1.345 & 0.839 & -1.529 & 0.998 & 0.955 & 0.265 & 0.891 & 0.436 \\
\hline & \multirow{4}{*}{$\begin{array}{l}\text { Right first } \\
\text { premolar }\end{array}$} & $R-L$ & 0.032 & 0.725 & -0.001 & 0.768 & 0.975 & 0.750 & 0.962 & 0.864 \\
\hline & & $A-P$ & 1.323 & 0.906 & 1.345 & 1.005 & 0.913 & 0.694 & 0.949 & 0.783 \\
\hline & & S-I & -0.853 & 0.803 & -0.794 & 0.877 & 0.883 & -0.052 & 0.906 & 0.037 \\
\hline & & $3 D$ & 1.882 & 0.952 & 1.937 & 1.015 & 0.924 & 0.702 & 0.949 & 0.835 \\
\hline & $\begin{array}{l}\text { Right } \\
\text { canine }\end{array}$ & $3 D$ & 1.661 & 0.651 & 1.590 & 0.737 & 0.867 & 0.476 & 0.946 & 0.611 \\
\hline & \multirow{4}{*}{$\begin{array}{c}\text { Central } \\
\text { incisor }\end{array}$} & $R-L$ & 0.090 & 0.424 & 0.089 & 0.502 & 0.716 & 0.050 & 0.869 & 0.332 \\
\hline & & $A-P$ & 0.284 & 0.656 & 0.332 & 0.619 & 0.949 & 0.607 & 0.957 & 0.777 \\
\hline & & S-I & -0.716 & 1.105 & -0.282 & 1.084 & 0.873 & 0.183 & 0.929 & 0.283 \\
\hline & & $3 D$ & 1.393 & 0.682 & 1.171 & 0.777 & 0.932 & 0.017 & 0.888 & -0.058 \\
\hline & \multirow{4}{*}{$\begin{array}{l}\text { Left } \\
\text { canine }\end{array}$} & $R-L$ & 0.175 & 0.732 & 0.201 & 0.810 & 0.921 & 0.583 & 0.972 & 0.668 \\
\hline & & $A-P$ & 0.774 & 0.972 & 0.898 & 0.848 & 0.946 & 0.627 & 0.956 & 0.754 \\
\hline & & $S-1$ & -0.830 & 1.085 & -0.562 & 0.986 & 0.939 & 0.018 & 0.774 & 0.023 \\
\hline & & $3 D$ & 1.793 & 0.849 & 1.688 & 0.780 & 0.944 & 0.628 & 0.938 & 0.475 \\
\hline & \multirow{2}{*}{$\begin{array}{l}\text { Left first } \\
\text { molar }\end{array}$} & S-I & -1.440 & 1.243 & -1.494 & 1.203 & 0.929 & 0.429 & 0.934 & 0.519 \\
\hline & & $3 D$ & 2.474 & 1.244 & 2.518 & 1.251 & 0.950 & 0.782 & 0.974 & 0.846 \\
\hline \multirow[t]{12}{*}{ T2-T3 } & \multirow{4}{*}{$\begin{array}{l}\text { Right first } \\
\text { molar }\end{array}$} & $R-L$ & -0.224 & 0.963 & -0.206 & 0.821 & 0.959 & 0.761 & 0.955 & 0.801 \\
\hline & & $A-P$ & 1.309 & 1.685 & 1.341 & 1.556 & 0.947 & 0.719 & 0.592 & 0.402 \\
\hline & & $S-1$ & -0.386 & 1.336 & -0.440 & 1.471 & 0.924 & 0.490 & 0.855 & 0.370 \\
\hline & & $3 D$ & 2.378 & 1.314 & 2.438 & 1.134 & 0.836 & 0.433 & 0.394 & 0.180 \\
\hline & \multirow{4}{*}{$\begin{array}{l}\text { Right first } \\
\text { premolar }\end{array}$} & $\mathrm{R}-\mathrm{L}$ & -0.449 & 0.649 & -0.468 & 0.535 & 0.956 & 0.621 & 0.748 & 0.068 \\
\hline & & A-P & 0.487 & 1.502 & 0.588 & 1.376 & 0.913 & 0.702 & 0.894 & 0.433 \\
\hline & & $S-I$ & -0.585 & 1.281 & -0.784 & 1.409 & 0.965 & 0.420 & 0.897 & 0.470 \\
\hline & & $3 D$ & 1.939 & 1.128 & 2.055 & 1.027 & 0.866 & 0.153 & 0.685 & 0.039 \\
\hline & \multirow{4}{*}{$\begin{array}{l}\text { Right } \\
\text { canine }\end{array}$} & $R-L$ & -0.267 & -0.709 & 0.382 & 0.671 & 0.652 & 0.490 & 0.673 & 0.264 \\
\hline & & $A-P$ & 0.181 & 1.212 & 0.208 & 1.229 & 0.869 & 0.585 & 0.909 & 0.289 \\
\hline & & S-I & -0.730 & 1.382 & -1.037 & 1.461 & 0.981 & 0.518 & 0.904 & 0.561 \\
\hline & & $3 D$ & 1.870 & 0.979 & 2.021 & 1.100 & 0.927 & 0.104 & 0.828 & 0.270 \\
\hline
\end{tabular}


TABLE 1 (Continued)

\begin{tabular}{|c|c|c|c|c|c|c|c|c|c|}
\hline & \multirow[b]{2}{*}{ Measurement } & \multicolumn{2}{|c|}{$\begin{array}{l}\text { CHANGES LA } \\
(\mathrm{mm})\end{array}$} & \multicolumn{2}{|c|}{$\begin{array}{l}\text { CHANGES ROI } \\
(\mathrm{mm})\end{array}$} & \multirow{2}{*}{$\begin{array}{l}\text { Intra-exam- } \\
\text { iner } \\
\text { agreement } \\
\text { LA }\end{array}$} & \multirow{2}{*}{$\begin{array}{l}\text { Inter-ex- } \\
\text { aminer } \\
\text { agreement } \\
\text { LA }\end{array}$} & \multirow{2}{*}{$\begin{array}{l}\text { Intra-exam- } \\
\text { iner } \\
\text { agreement } \\
\text { ROI }\end{array}$} & \multirow{2}{*}{$\begin{array}{l}\text { Inter-exam- } \\
\text { iner } \\
\text { agreement } \\
\text { ROI }\end{array}$} \\
\hline & & Mean & SD & Mean & SD & & & & \\
\hline \multirow{4}{*}{$\begin{array}{l}\text { Central } \\
\text { incisor }\end{array}$} & $\mathrm{R}-\mathrm{L}$ & 0.081 & 0.759 & -0.044 & 0.726 & 0.466 & 0.199 & 0.780 & 0.179 \\
\hline & $A-P$ & -0.215 & 1.320 & -0.260 & 1.282 & 0.960 & 0.852 & 0.984 & 0.745 \\
\hline & S-I & -0.963 & 1.631 & -1.245 & 1.702 & 0.966 & 0.580 & 0.978 & 0.580 \\
\hline & $3 D$ & 2.175 & 1.068 & 2.287 & 1.172 & 0.896 & 0.237 & 0.966 & 0.155 \\
\hline \multirow{4}{*}{$\begin{array}{l}\text { Left } \\
\text { canine }\end{array}$} & $\mathrm{R}-\mathrm{L}$ & -0.217 & 0.845 & -0.172 & 0.864 & 0.812 & 0.458 & 0.849 & 0.376 \\
\hline & $A-P$ & 0.016 & 1.064 & -0.151 & 1.002 & 0.634 & 0.417 & 0.874 & 0.520 \\
\hline & S-I & -1.018 & 1.418 & -0.910 & 1.637 & 0.942 & 0.559 & 0.920 & 0.510 \\
\hline & $3 D$ & 1.499 & 1.364 & 1.969 & 1.169 & - & 0.029 & 0.832 & 0.181 \\
\hline \multirow{4}{*}{$\begin{array}{l}\text { Left first } \\
\text { premolar }\end{array}$} & $\mathrm{R}-\mathrm{L}$ & -0.421 & 0.925 & -0.287 & 0.757 & - & 0.066 & 0.874 & 0.470 \\
\hline & $A-P$ & 0.628 & 1.354 & 0.421 & 1.273 & 0.214 & 0.593 & 0.922 & 0.470 \\
\hline & S-I & -0.779 & 1.183 & -0.562 & 1.445 & 0.932 & 0.421 & 0.896 & 0.461 \\
\hline & $3 D$ & 1.906 & 1.127 & 1.866 & 1.145 & 0.748 & 0.105 & 0.787 & 0.277 \\
\hline \multirow{4}{*}{$\begin{array}{l}\text { Left first } \\
\text { molar }\end{array}$} & $R-L$ & 0.072 & 0.749 & 0.032 & 0.793 & 0.899 & 0.715 & 0.984 & 0.653 \\
\hline & $A-P$ & 0.970 & 1.282 & 0.826 & 1.308 & 0.697 & 0.311 & 0.809 & 0.236 \\
\hline & S-I & -0.485 & 1.333 & 0.091 & 1.815 & 0.918 & 0.390 & 0.888 & 0.423 \\
\hline & $3 D$ & 1.962 & 1.118 & 2.141 & 1.284 & 0.768 & 0.173 & 0.611 & 0.163 \\
\hline
\end{tabular}

R-L, right-left plane; A-P, anteroposterior plane; S-I, superoinferior plane; 3D, three-dimensional.

inter-examiner agreements for LA and ROI registration methods were calculated using intraclass correlation coefficient (ICC) and Bland-Altman limits of agreement. ${ }^{19}$ ICC values from 0.75 to 1 , from 0.6 to 0.74 , from 0.4 to 0.59 and less than 0.4 were considered, excellent, good, fair and poor agreements, respectively. ${ }^{20}$

\section{3 | RESULTS}

Table 1 shows the intra- and inter-examiner agreements. For both superimposition methods, excellent or good intra-examiner agreement was found for most T1-T2 and T2-T3 measurements (Table 1). Inter-examiner agreements were good for T1-T2 measurements and poor to fair for most T2-T3 measurements for both LA and ROI superimposition methods (Table 1 ).

Mean T1-T2 differences were less than $0.5 \mathrm{~mm}$ for almost all variables (Tables 2 and 3). Most of the intra-examiner error limits ranged from -0.5 to $+0.5 \mathrm{~mm}$ in T1-T2 interval and from -1 to $+1 \mathrm{~mm}$ in T2-T3 interval for both LA and ROI superimposition methods (Tables 2 and 3). Most of the inter-examiner lower and upper error limits ranged from -2 to $+2 \mathrm{~mm}$ in T1-T2 interval and from -2.5 to $+2.5 \mathrm{~mm}$ in T2-T3 interval, similarly in both superimposition methods (Tables 2 and 3 ).

\section{4 | DISCUSSION}

Palatal rugae have been described as relatively stable references during facial growth, mainly in the sagittal and transversal planes. ${ }^{8}$
The distal limit of the incisive papilla and the medial point of palatal rugae have been reported as being more stable during both growth and orthodontic treatment. ${ }^{2,10,11}$ The medial and lateral limit of the third rugae also has been shown to be reliable due to its positional stability. ${ }^{2,10,11}$ On the other hand, the lateral limit of the first and second rugae points seems to change following maxillary tooth movements. ${ }^{8}$ However, no previous studies evaluated the reliability of palatine rugae in a 40-year interval. Adipose tissue decreases over ageing and might affect rugae volume and definition. ${ }^{21}$

For this study, 5 landmarks were placed at stable regions, including the posterior limit of incisive papilla and the medial end of second and third rugae. Additionally, two landmarks were placed at the lateral end of third rugae to avoid the rotation of dental models in the coronal plane (roll) during registration. The landmarks placed $10 \mathrm{~mm}$ posteriorly to the third rugae were used to avoid the rotation of dental models in the sagittal plane (pitch) during superimposition.

The superimposition method using anatomical landmarks demonstrated reliability for evaluating dental changes within a 5-year period with good to excellent intra- and inter-examiner agreements for most measurements and a small range of error. Previous studies also found that palatal rugae were suitable for maxillary model superimpositions. ${ }^{2,3,10}$ On the other hand, within a time interval of 42 years, the reproducibility of dental changes measurements was inadequate in view of the inter-examiner fair to poor agreements.

Interestingly, dental changes per unit of time were rather larger in T1-T2 interval compared to T2-T3 interval. Therefore, the size of error from 13 to 18 years was not related directly to the amount of dental changes occurring, which was substantial. On the other hand, 


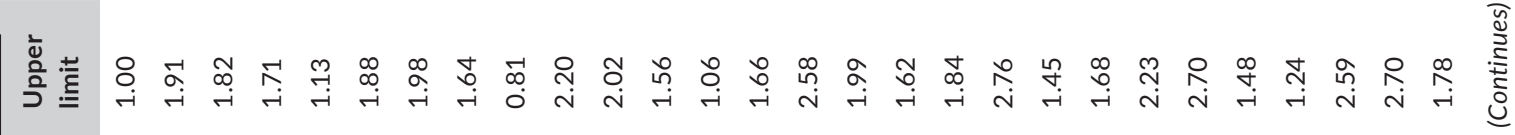

产苞

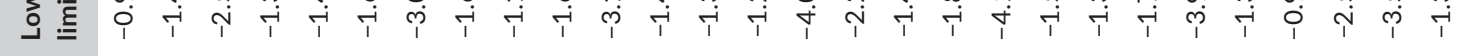

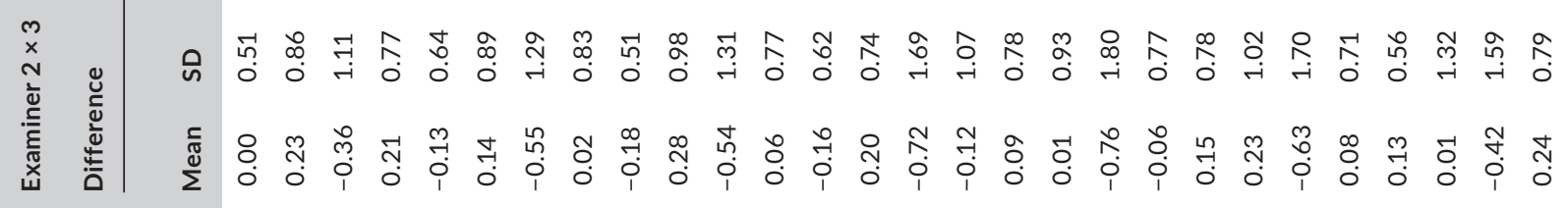

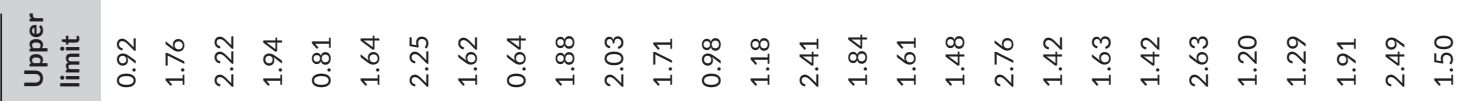

商苟

刍血

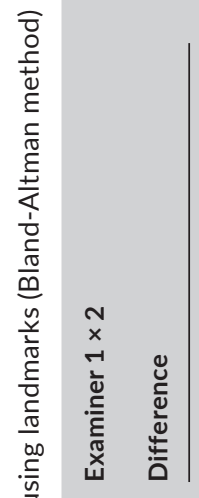

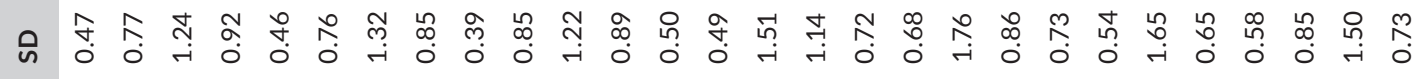

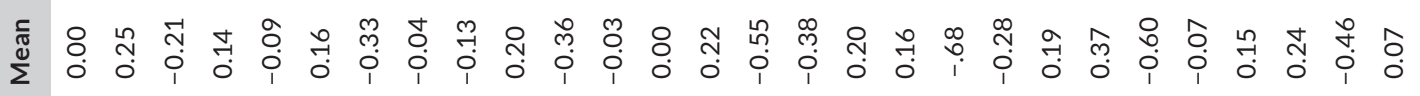

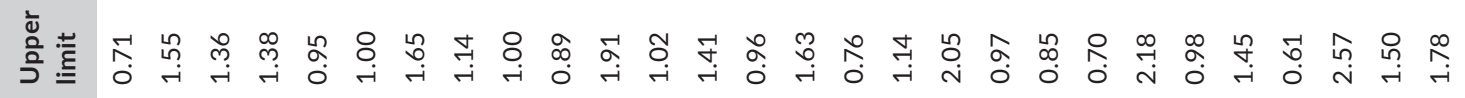

乡ँ๊

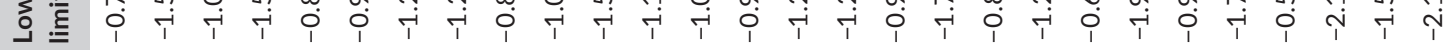

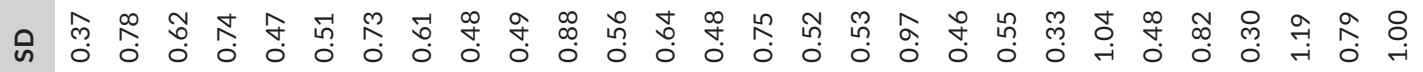

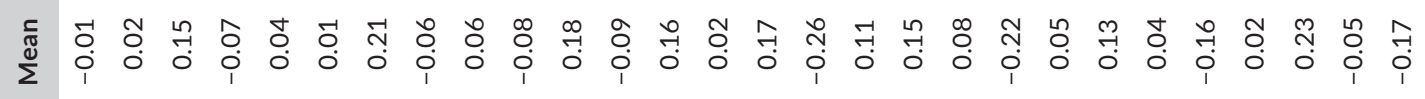

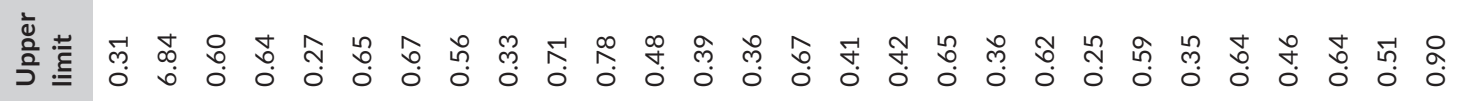

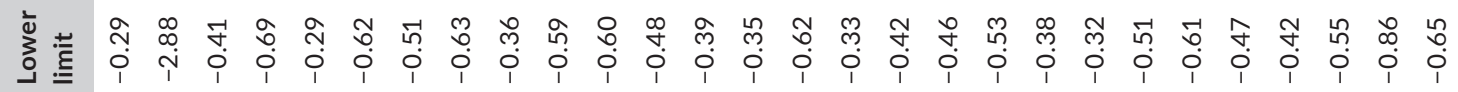

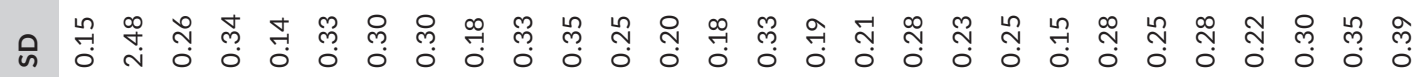

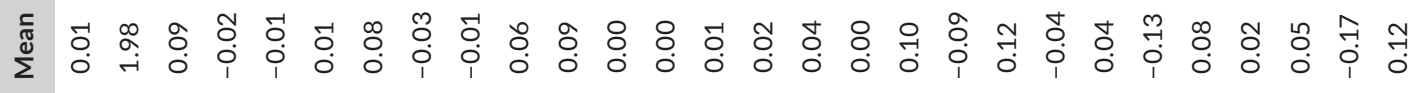

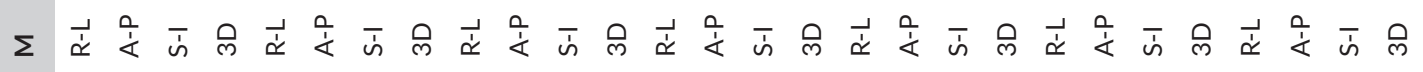

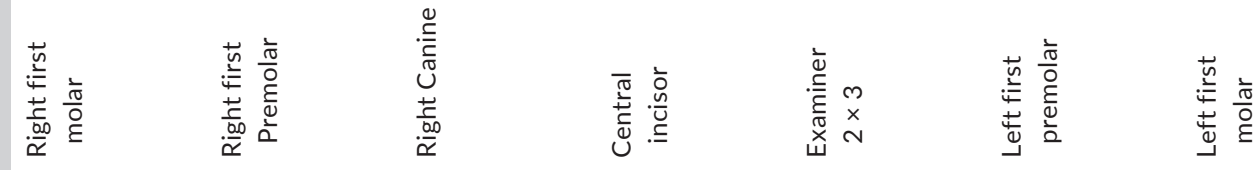




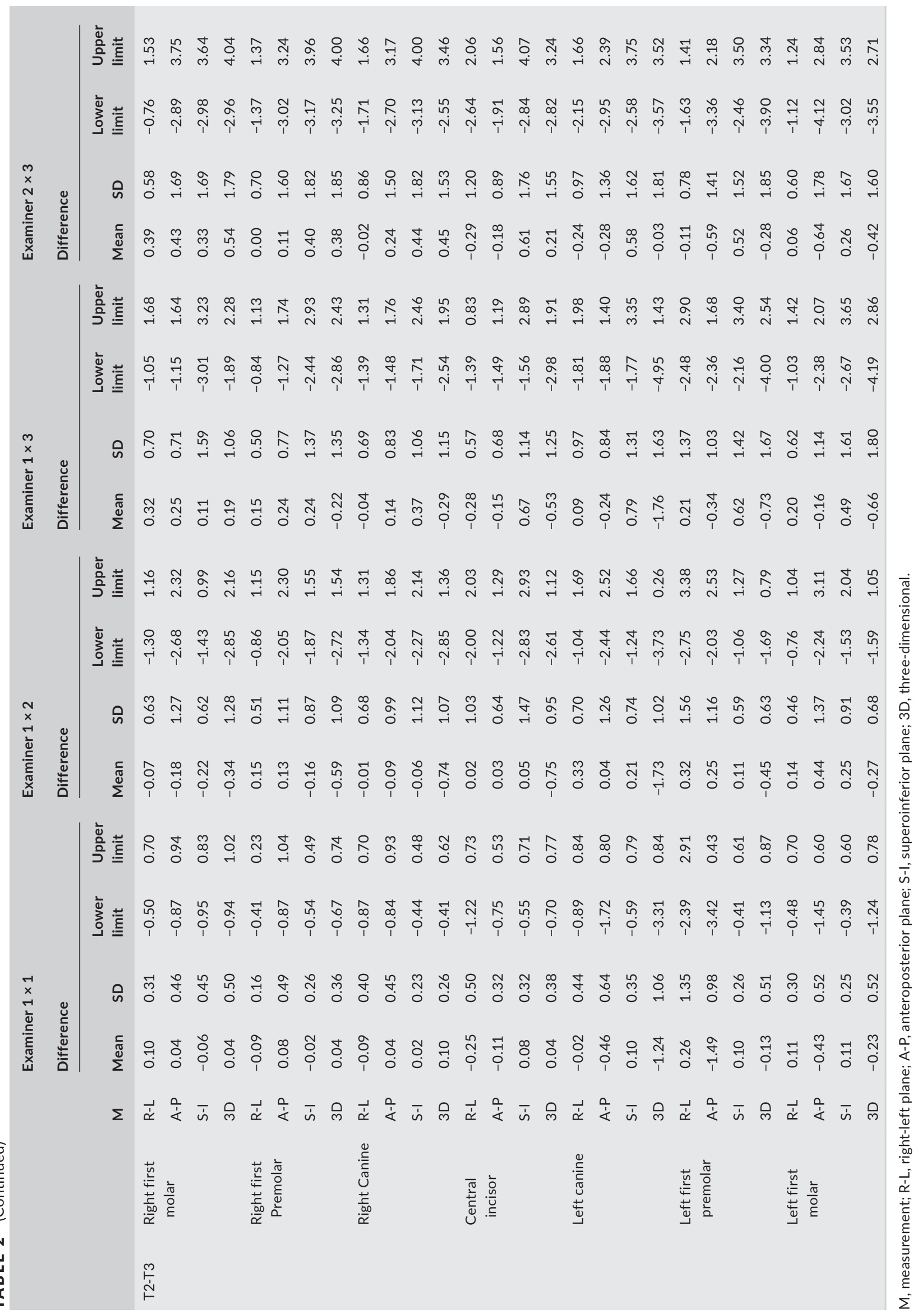




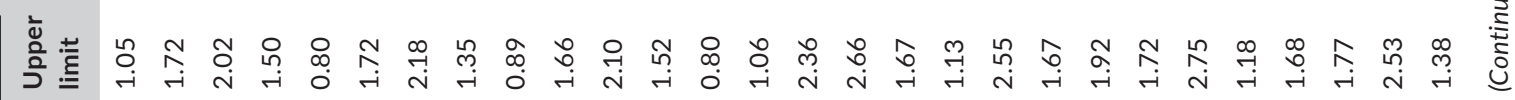

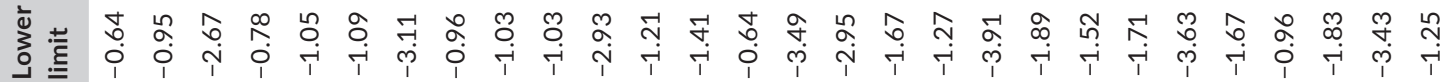

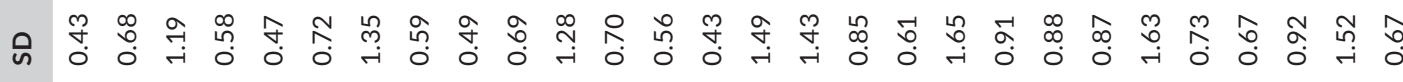

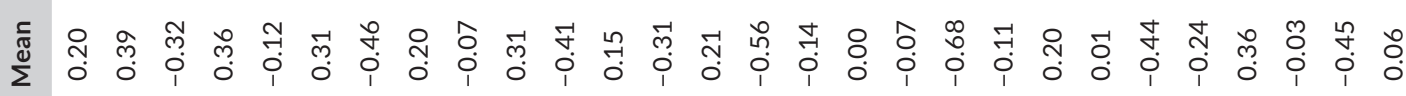

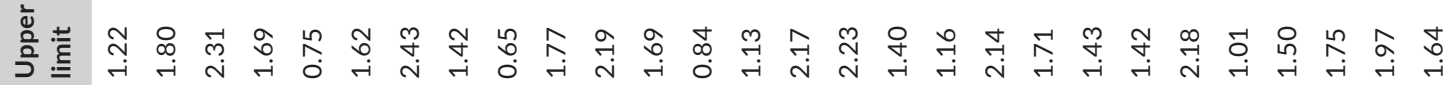

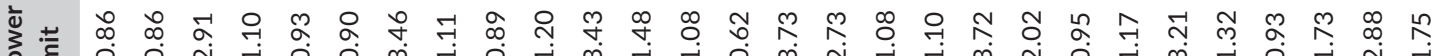
3.

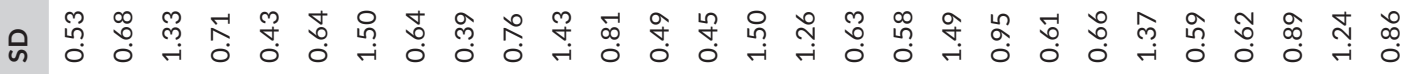

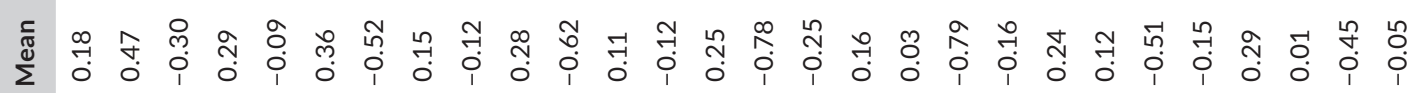

商芴

产节

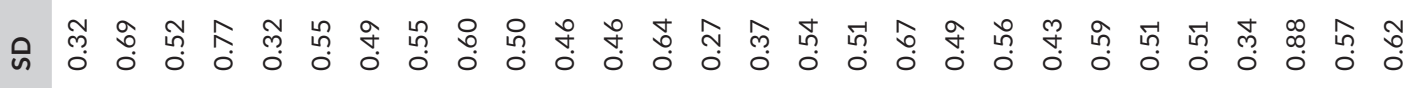

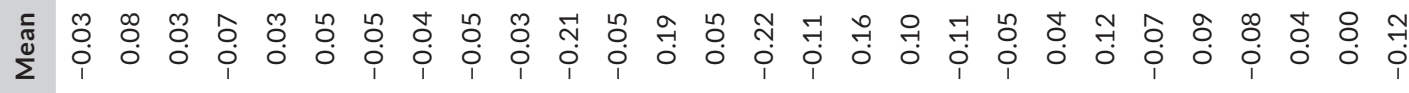

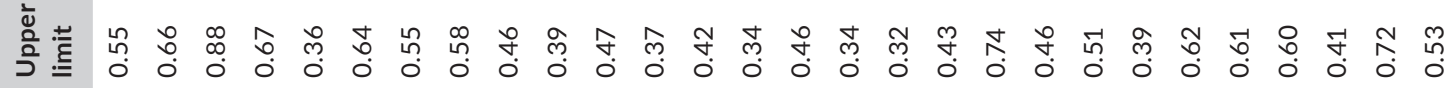

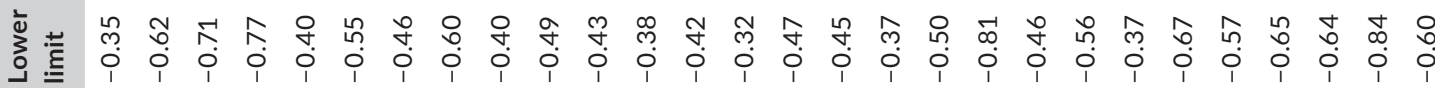

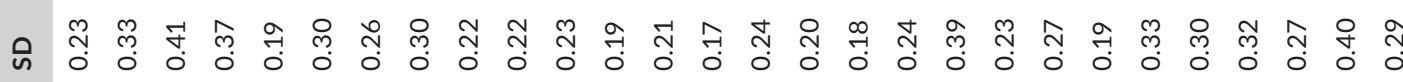

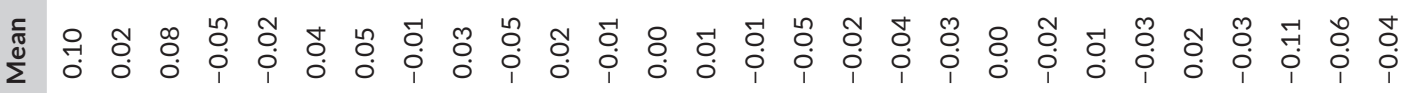

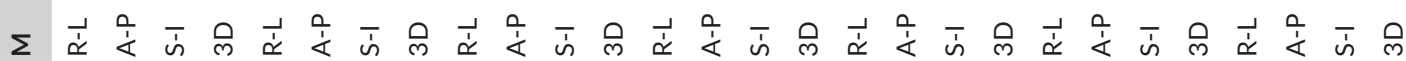

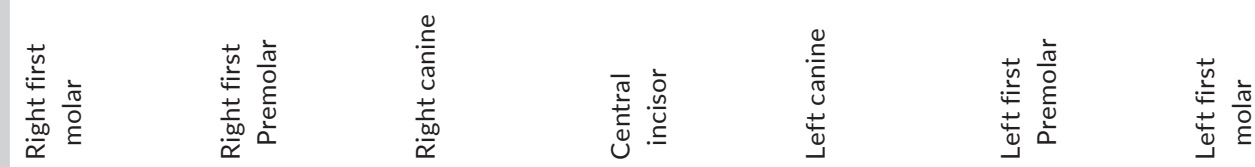

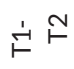




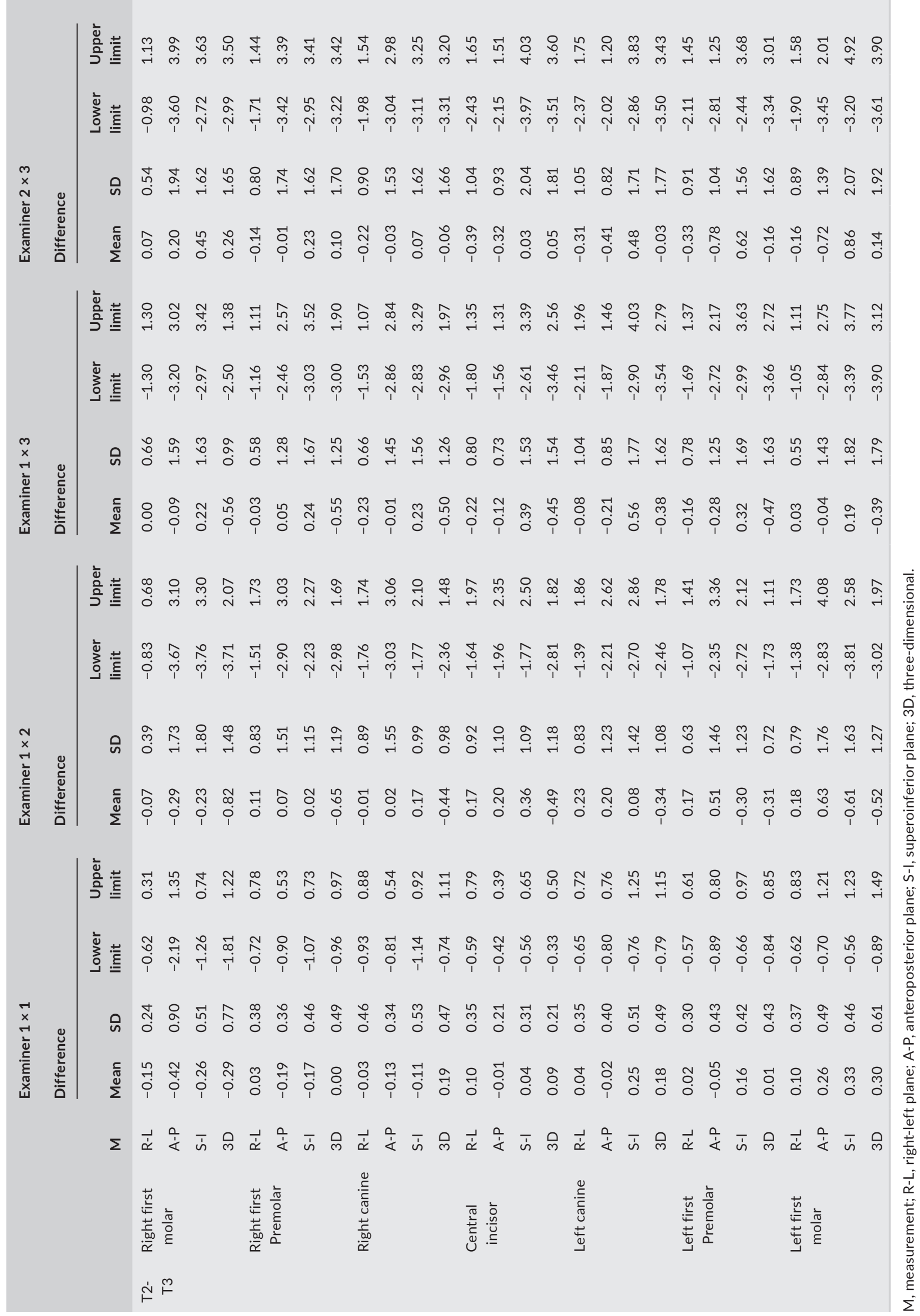


from 18 to 60 years of age, dental changes were very small per unit of time and the error then was consequential.

All three examiners faced difficulties identifying rugae landmarks in T3 models due to three reasons: a slight elongation of the lateral termination of the third rugae; some loss of volume and definition of the palatal rugae with ageing; and missing anatomical landmarks in some impressions. Therefore, palatal rugae seem to be unreliable when the time interval for model registration corresponds to decades instead of years.

Similar levels of agreement and error limits were found for registration using regions of interest as were found using anatomical landmarks. The use of regions of interest for computation of consecutive dental model registration has not produced a more reliable method of measuring changes in the dentition over time. There was some similarity between LA and ROI registration for measurements that demonstrated high inter-examiner errors from T2 to T3. These results were expected in that the definition of the region of interest is dependent of the same landmark identification used for point of interest registration. Therefore, errors in the identification landmark might decrease the reliability of LA and ROI registration methods.

Considering the similar adequate reproducibility found from T1 to $\mathrm{T} 2$ for both LA and ROI registrations, the authors assume registration based on landmarks may be the preferable method for this untreated sample. This approach also may be useful in the evaluation of patients undergoing orthodontic treatment as long as the location of the landmarks has not been affected by intervention involving arch expansion or closure of extraction spaces. Considering the digital model surface mesh is organized differently in each time point, a same radius of regions of interest produced different size areas in T1, T2 and T3 dental models. These differences sometimes produced some changes in the degree of pitch, roll and yaw (Figure 1) in the registered model. A solution to overcome these problems would be to "remesh" or normalize the surface meshes before registration, as open source software and commercial tools allow. But even if the meshes were more regular, the different time points do not have exactly the same corresponding meshes.

Registration of digital models allows assessments of individual tooth movement rather than overall skeletal displacement of the craniofacial complex, as registration relative to the cranial base allows. Using metallic implants, Gu and McNamara ${ }^{22}$ have found that the lingual curvature of the hard palate shows apposition and downward displacement during growth. We assume that palatal rugae follow hard palate changes. Therefore, the superimposition on the palatine rugae might underestimate the vertical movements of the maxillary dentition. This underestimation is a limitation of registering digital dental models on the palatal rugae. For this reason, researchers and clinicians should carefully interpret measurements relative to structures of reference used.

The evaluation of three-dimensional tooth movement during long-term ageing process was not reliable using palatal rugae registration due to low inter-examiner agreements. Short-term assessment of three-dimensional dental changes using palatal rugae registration that was based both on landmarks or regions of interest showed adequate reproducibility. When using this method, dental changes should be described relatively to the palatal rugae. Future studies using registration of cone-beam computed tomography and digital dental model images should evaluate the accuracy of maxillary dental model superimposition.

\section{5 | CONCLUSION}

Maxillary digital dental models of patients with normal occlusion superimposed on palatal rugae for measuring three-dimensional dental changes over a 5-year interval showed an adequate reliability using both landmarks and regions of interest. On the other hand, 40-year interval measurements using both superimposition methods showed lower than acceptable reproducibility.

\section{CONFLICT OF INTEREST}

The authors have no conflicts of interest to declare.

\section{ORCID}

Daniela Garib (iD https://orcid.org/0000-0002-2449-1620

Marilia S. Yatabe iD https://orcid.org/0000-0002-8748-6714

\section{REFERENCES}

1. Ganzer N, Feldmann I, Liv P, Bondemark L. A novel method for superimposition and measurements on maxillary digital 3D modelsstudies on validity and reliability. Eur J Orthod. 2018;40(1):45-51.

2. Choi JI, Cha BK, Jost-Brinkmann PG, Choi DS, Jang IS. Validity of palatal superimposition of 3-dimensional digital models in cases treated with rapid maxillary expansion and maxillary protraction headgear. Korean J Orthod. 2012;42(5):235-241.

3. Miller RJ, Kuo E, Choi W. Validation of Align Technology's Treat III digital model superimposition tool and its case application. Orthod Craniofac Res. 2003;6(1):143-149.

4. Thiruvenkatachari B, Al-Abdallah M, Akram NC, Sandler J, O'Brien $\mathrm{K}$. Measuring 3-dimensional tooth movement with a 3-dimensional surface laser scanner. Am J Orthod Dentofacial Orthop. 2009;135(4):480-485.

5. Talaat S, Kaboudan A, Bourauel C, Ragy N, Kula K, Ghoneima A. Validity and reliability of three-dimensional palatal superimposition of digital dental models. Eur J Orthod. 2017;39(4):365-370.

6. Cevidanes LH, Heymann G, Cornelis MA, DeClerck HJ, Tulloch JF. Superimposition of 3-dimensional cone-beam computed tomography models of growing patients. Am J Orthod Dentofacial Orthop. 2009;136(1):94-99.

7. Houston WJ, Lee RT. Accuracy of different methods of radiographic superimposition on cranial base structures. Eur J Orthod. 1985;7(2):127-135.

8. Almeida MA, Phillips C, Kula K, Tulloch C. Stability of the palatal rugae as landmarks for analysis of dental casts. Angle Orthod. 1995;65(1):43-48.

9. Bailey LT, Esmailnejad A, Almeida MA. Stability of the palatal rugae as landmarks for analysis of dental casts in extraction and nonextraction cases. Angle Orthod. 1996;66(1):73-78. 
10. Hoggan BR, Sadowsky C. The use of palatal rugae for the assessment of anteroposterior tooth movements. Am J Orthod Dentofacial Orthop. 2001;119(5):482-488.

11. Jang I, Tanaka M, Koga Y, et al. A novel method for the assessment of three-dimensional tooth movement during orthodontic treatment. Angle Orthod. 2009;79(3):447-453.

12. Simmons JD, Moore RN, Erickson LC. A longitudinal study of anteroposterior growth changes in the palatine rugae. J Dent Res. 1987;66(9):1512-1515.

13. Choi DS, Jeong YM, Jang I, Jost-Brinkmann PG, Cha BK. Accuracy and reliability of palatal superimposition of three-dimensional digital models. Angle Orthod. 2010;80(4):497-503.

14. Behrents RG. A treatise on the continuum of growth in the aging craniofacial skeleton Monograph 17, Craniofacial Growth Series, Center for Human Growth and Development, The University of Michigan. 1985.

15. Carter GA, McNamara JA Jr. Longitudinal dental arch changes in adults. Am J Orthod Dentofacial Orthop. 1998;114(1):88-99.

16. Sinclair PM, Little RM. Maturation of untreated normal occlusions. Am J Orthod. 1983;83(2):114-123.

17. Thilander B. Dentoalveolar development in subjects with normal occlusion. A longitudinal study between the ages of 5 and 31 years. Eur J Orthod 2009;31:109-120.
18. Massaro C, Miranda F, Janson G, et al. Maturational changes of the normal occlusion: a 40-year follow-up. Am J Orthod Dentofacial Orthop. 2018;154(2):188-200.

19. Bland JM, Altman D. Statistical methods for assessing agreement between two methods of clinical measurement. Lancet. 1986;327(8476):307-310.

20. Fleiss JL. Reliability of Measurement. The Design and Analysis of Clinical Experiments. New York, NY: John Wiley \& Sons, Inc.; 1999:1-32.

21. Gierloff M, Stohring C, Buder T, Gassling V, Acil Y, Wiltfang J. Aging changes of the midfacial fat compartments: a computed tomographic study. Plast Reconstr Surg. 2012;129(1):263-273.

22. Gu Y, McNamara JA Jr. Cephalometric superimpositions: a comparison of anatomical and metallic implant methods. Angle Orthod. 2008;78(6):967-976.

How to cite this article: Garib D, Miranda F, Yatabe MS, et al. Superimposition of maxillary digital models using the palatal rugae: Does ageing affect the reliability?. Orthod Craniofac Res. 2019;22:183-193. https://doi.org/10.1111/ocr.12309 